\title{
Range-Spread Target Adaptive Detector for Non-Gaussian Clutter
}

\author{
Gu Xinfeng ${ }^{1, ~ *, ~ H a o ~ X i a o l i n ~}{ }^{2}$, Liu Tongling ${ }^{1}$, Yang Ganlin ${ }^{1}$, Li Xinxing ${ }^{1}$ \\ ${ }^{1}$ China Satellite Maritime Tracking \& Control Department, Jiangyin, China \\ ${ }^{2}$ Yantai Electricity and Economy Technical Institute, Yantai, China
}

\section{Email address:}

y6ckgxf@126.com (Gu Xinfeng)

${ }^{*}$ Corresponding author

\section{To cite this article:}

Gu Xinfeng. Range-Spread Target Adaptive Detector for Non-Gaussian Clutter. Science Discovery. Vol. 5, No. 1, 2017, pp. 48-52. doi: $10.11648 /$ j.sd.20170501.18

Received: February 22, 2017; Accepted: March 22, 2017; Published: April 12, 2017

\begin{abstract}
This paper addresses range-spread target detection in non-Gaussian clutter modeled as spherically invariant random vector clutter (SIRV). A adaptive modified scatterer density dependent (AMSDD) detector based on clutter power is addressed for the problem that MSDD has detection loss when falsely estimating the number of scatterers. Firstly, it estimates the scatterers by the power of clutter. Then, the returns of target scatterers are used to compare with the threshold to determine whether the target is exist or not. Compare with the MSDD and the NSDD, the AMSDD, adaptively estimates the number of target scatterers according to the observations, which can improve performance and robustness of detector effectively.
\end{abstract}

Keywords: Non-Gaussian Clutter, Range-Spread Rarget, Detection, Adaptive

\section{非高斯背景下距离扩展目标的自适应检测器}

顾新锋 ${ }^{1 *}$, 郝晓琳 ${ }^{2}$, 刘童岭 ${ }^{1}$, 杨甘霖 $^{1}$, 李新星 ${ }^{1}$

${ }^{1}$ 中国卫星海上测控部, 江阴, 中国

${ }^{2}$ 烟台电力经济技术研究所, 烟台, 中国

邮箱

y6ckgxf@126. com（顾新锋）

摘要：本文研究了采用SIRV建模的非高斯杂波背景下的距离扩展目标检测问题。针对MSDD检测器在估计目标散射点个 数估计失配时存在一定检测损失的问题，提出了一种基于杂波功率的自适应MSDD检测器（AMSDD）。AMSDD首先利用杂 波功率估计目标散射点个数, 再利用目标散射点回波进行积累并与國值比较, 判断目标有无。AMSDD能根据实际观测值 自适应的估计目标散射点个数，与MSDD和NSDD相比AMSDD具有更好的检测性能和鲁棒性。

关键词: 非高斯杂波, 距离扩展目标, 检测, 自适应 


\section{1. 引言}

与低分辨率雷达不同，高分辨率雷达能够在空间上 把一个目标分解成许多散射点, 目标回波在雷达径向上 的多个强散射点分布在不同的距离单元中, 形成距离扩 展目标 $[1,2]$ 。目标的离散散射中心常被称为多主散射中 心MDS (multiple dominant scattering)。增加雷达分 辨率并采用适当的检测方法能极大地提高检测概率 [3], 但如果采用传统点目标检测器, 由于部分目标能量泄漏 到参考单元中, 检测性能将大大下降, 甚至完全失效 [4]。 文献 [5-7]研究了高斯杂波背景下距离扩展目标的检测 方法, 但在高分辨率情况下, 雷达会接收到类似于目标 的尖峰, 此时高斯模型不再适用, 这种含尖峰的杂波可 用球不变随机向量 SIRV (spherically invariant random vector) 来建模 $[2,8]$ 。文献 [9]在SIRV杂波条件 下, 提出了不依赖于散射点密度的广义似然比检验检测 器NSDD (non-scatterer density dependent) 和基于目 标散射点密度先验信息的检测器 SDD (scatterer density dependent)。由于SDD是统计平均意义下的最优 检测, 未能充分利用散射点密度的先验信息, 存在一定 的检测损失。文献 [10]有效利用目标散射点信息, 获得 了修正的检测器MSDD (modified SDD), 证明了MSDD的恒 虚警率 (CFAR, constant false alarm rate)特性。虽然 MSDD的检测性能要优于NSDD和SDD, 但估计的目标散射点 个数实际值失配时, MSDD仍存在一定的检测损失。文献 [11]采用 $\mathrm{AD}$ 检验的方法估计散射点个数, 可以提高信杂 比, 但未能考虑实际的观测情况的起伏变化, 为此, 本 文提出了自适应的MSDD检测器AMSDD (Adaptive MSDD), 根据实际观测值估计散射点个数, 并动态调整检测阈值, 提高了检测性能, 增强了系统的鲁棒性。

\section{2. 问题描述}

假设数据从 $N$ 个阵元得到, 需解决跨过 $K$ 个距离单元 $Z_{t}$, $t=1,2, \cdots, K$ 检测一个目标存在与否的问题。假设可能的目 标完全包含在这 $K$ 个距离单元中, 并且忽略距离走动的问 题。考虑杂波为主的干扰环境, 忽略内部噪声的影响。所 要解决的检测问题可表示为:

$$
\begin{aligned}
& \mathrm{H}_{0}: \mathrm{z}_{t}=\mathrm{c}_{t}, \quad t=1, \ldots, K \\
& \mathrm{H}_{1}: \mathrm{z}_{t}=\alpha_{t} \mathrm{p}+\mathrm{c}_{t}, t=1, \ldots, K
\end{aligned}
$$

式中, $p$ 表示已知的单位导向矢量, 即 $p^{H} p=1$ （符号 “ ( ・ $)^{\mathrm{H} ”}$ 表示共轭转置); 标量 $a_{t}$ 是反映目标和信道影响 的未知确定性参数。当 $\mathrm{p}=\left(1, e^{j \varphi}, e^{j 2 \varphi}, \ldots, e^{j(N-1) \varphi}\right)^{\mathrm{T}} / \sqrt{N}$ 时 (其中 $\varphi$ 为常数, 符号 “ $(\cdot)^{\mathrm{T}}$ ” 表示转置), 式 (1) 表示 的目标模型与移动距离扩展目标模型相似 [9], 在那里假 设目标在 $N$ 个脉冲的相干处理时间内无距离走动。

杂波回波用 SIRV 分布来建模 [5], 则杂波向量 $\boldsymbol{c}_{t}$ 可以表示成

$$
\mathrm{c}_{t}=\sqrt{\tau_{t}} \cdot \eta_{t}, \quad t=1, \ldots, K
$$

式中, $\quad \eta_{t}=\left(\eta_{t}(1), \quad \eta_{t}(2), \cdots, \quad \eta_{t}(K)\right)^{\mathrm{T}}, \quad \eta_{t}(n)$, $n=1,2, \cdots, N$ 是独立同分布 (IID, independent and identically distributed) 的零均值方差为 1 的复圆高斯 随机变量; 纹理分量 $\tau_{t}$ 是服从分布 $f_{\tau}$ 的非负随机变量, 用来描述不同距离单元间杂波功率水平的起伏。根据SIRV 模型可知, $\eta_{t}$ 和 $\tau_{t}$ 是相互独立的, 且 $\eta_{t}$ 的归一化协方差 矩阵 $\Sigma$ 表示为

$$
\Sigma=E\left(\eta_{t} \eta_{t}^{\mathrm{H}}\right), \quad t=1, \ldots, K
$$

其中 $\Sigma$ 为正定的Hermitian矩阵。

为了简化模型, 假设一个距离单元最多只有一个散射 点, 即目标所占距离单元个数与目标散射点个数相同。令 集合 $\Theta_{h_{0}}$ 表示存在目标散射点的距离单元下标的集合, $h_{0}$ 表示目标散射点所占距离单元个数, $h_{\mathrm{e}}$ 表示 $h_{0}$ 的估计值。 在未知 $a_{t}$ 和 $f_{\tau}$ 的条件下, 基于有序统计理论, 对SDD进行 修正, 可得MSDD的检测统计量为 [10]

$$
\lambda_{\mathrm{MSDD}}=-2(N-1) \sum_{t \in \Theta_{h_{0}}} \ln \left(1-w_{t}\right)
$$

其中,

$$
w_{t}=\frac{\left|\mathrm{p}^{\mathrm{H}} \Sigma^{-1} \mathrm{z}_{t}\right|^{2}}{\left(\mathrm{z}_{t}^{\mathrm{H}} \Sigma^{-1} \mathrm{z}_{t}\right)\left(\mathrm{p}^{\mathrm{H}} \Sigma^{-1} \mathrm{p}\right)}, \quad t=1, \ldots, K
$$

将 $w_{t}$ 按从大到小的顺序排列, 可得有序序列 $1 \geqslant W_{(1)} \geqslant_{W_{(2)}} \geqslant \cdot \cdot \cdot \geqslant_{W_{(K)}} \geqslant 0$ 。利用 $W_{(h)}, k=1,2, \cdots, K$ 中最 大的 $h_{0}$ 个值进行积累可得MSDD检验统计量为:

$$
\lambda_{\mathrm{MSDD}}=-2(N-1) \sum_{k=1}^{h_{0}} \ln \left(1-w_{(k)}\right)
$$

当 $h_{0}=K$ 时, MSDD退化为 $\mathrm{NSDD}^{[9]}$

$$
\lambda_{\mathrm{NSDD}}=-2(N-1) \sum_{k=1}^{K} \ln \left(1-w_{(k)}\right)
$$

\section{AMSDD检测器}

与SDD检测器相似, MSDD检测器需要事先估计目标散 射点数目, 当估计值与真实值不匹配时, 存在一定的检测 性能损失, 特别的当估计值 $h_{e}<h_{0}$ 时, 由于部分存在目标 散射点的距离单元没有被积累, MSDD存在较大的检测损失, 这一点将在后面的仿真实验中得到验证。本节将采用自适 应散射点估计方法, 增强检测器对未知散射点密度目标检 测的鲁棒性。

AMSDD检测器是这样工作的：首先根据雷达回波估计 目标强散射点个数, 然后对强散射点进行积累与相应的门 限进行比较判决目标有无。令 


$$
u_{t}=-2(N-1) \ln \left(1-w_{t}\right)
$$

由于函数 $g(x)=-2(N-1) \ln (1-x)$ 在区间 $(0,1)$ 上是单 调增函数, 因此也可以通过 $u_{t}, t=1,2, \cdots, K$ 中最大的 $h_{0}$ 个 值来确定目标散射点位置的集合 $\Theta_{h_{0}}$ 。令 $u_{t}, \quad t=1,2, \cdots, K$ 的降序序列为 $u_{(1)} \geqslant u_{(2)} \geqslant \cdots \geqslant u_{(n)} \geqslant 0$, 在 $H_{0}$ 假设 下， $u_{t}$ 服从自由度为 2 的 $\chi^{2}$ 分布 [9] (也可认为是均值为 2 的指数分布), 当有强散射点存在时, $u_{t}$ 的均值将大于 2 , 因此, 可以分别计算 $h_{0}=1, \cdots, K$ 时序列中最小的 $K-h_{0}$ 个 回波的均值 $\mu_{h_{0}}$ 。从 $h_{0}=1$ 开始计算, 若 $\mu_{h_{0}} \geq 2$, 说明最 小的 $K-h_{0}$ 个回波中含有强散射点的回波，则 $h_{0}=h_{0}+1$; 若 $\mu_{h_{0}}<2$ 时, 认为强散射点已提取完毕, 停止计算, 取当 前的 $h_{0}$ 值作为强散射点个数的估计值 $h_{\mathrm{e}}$ 。由于序列是降序 排列的, 当 $h_{0}>h_{e}$ 时, 都有 $\mu_{h_{0}}<2$, 因此, 强散射点的估 计值可以表示为

$$
h_{\mathrm{e}}=\min _{h_{0}} \frac{1}{K-h_{0}} \sum_{k=h_{0}+1}^{K} u_{(k)}<2, \quad\left(0<h_{0}<K\right)
$$

式中, $u_{(1)} \geqslant u_{(2)} \geqslant \cdots \geqslant u_{(n)}$, 特别地, 若 $u_{(n)}>2$, 则 $h_{e}=K$ 。通过式 (9) 确定强散射点个数 $h_{\mathrm{e}}$, 对最大的 $h_{\mathrm{e}}$ 个 $u_{t}$ 进行积累检测, 则 AMSDD的检测统计量为:

$$
\lambda_{\mathrm{AMSDD}}=\sum_{k=1}^{h_{\mathrm{e}}} u_{(k)}
$$

由于AMSDD在本质上与MSDD具有一致性，因此由文献 [10]的分析可知, 与MSDD类似, AMSDD也具有CFAR特性。 当 $h_{0}=K$, 即目标散射点占据整个距离窗时, 式 (10) 等价 于式 (7), AMSDD退化为NSDD。比较式 (10) 和式 (6) 可知, AMSDD 与MSDD的主要区别在于 $h_{\mathrm{e}}$ 是根据实际的目标强散射 点密度自适应变化的, 不需要事先知道散射点密度的先验 信息, 提高了检测器对未知散射点先验信息目标检测的鲁 棒性。

\section{4. 性能分析}

本节对MSDD和AMSDD进行了比较分析。仿真中, 采用 $\Gamma$ 分布来表示混合分布 $f_{\tau}$

$$
f_{\tau}(\tau)=(L / b)^{L} \tau^{L-1} e^{-(L / b) \tau} / \Gamma(L), \quad \tau \geq 0
$$

式中 $\Gamma(\cdot)$ 是Gamma函数, $b$ 是均值, $L$ 用来控制与正态 统计量偏离的程度。

$K$ 个距离单元内每个距离单元的目标平均功率为 $\sigma_{s}^{2}$, 杂波平均功率为 $\sigma_{\mathrm{c}}^{2}$ 。有目标散射点的距离单元 $\left(t \in \Theta_{h_{0}}\right)$ 回波用方差为 $\varepsilon_{t} \sigma_{s}^{2} K$ 的独立零均值复圆高斯随机变量建 模, 其中 $\varepsilon_{t}$ 表示距离单元 $t$ 内的散射点能量占目标总能量 的比例。本文考虑几种典型的MDS模型, $\varepsilon_{t}$ 的具体取值如 表1所示。
表1 不同MDS模型的 $\varepsilon_{t}$ 值。

\begin{tabular}{lllll}
\hline 目标距离单元 & 1 & 2 & $\cdots$ & $h_{0}$ \\
\hline Mode 1.1 & $1 / h_{0}$ & $1 / h_{0}$ & $1 / h_{0}$ & $1 / h_{0}$ \\
Mode 1.2 & 0.50 & $0.50 /\left(h_{0}-1\right)$ & $0.50 /\left(h_{0}-1\right)$ & $0.50 /\left(h_{0}-1\right)$ \\
Mode 1.3 & 0.90 & $0.10 /\left(h_{0}-1\right)$ & $0.10 /\left(h_{0}-1\right)$ & $0.10 /\left(h_{0}-1\right)$ \\
Mode 1.4 & 0.99 & $0.01 /\left(h_{0}-1\right)$ & $0.01 /\left(h_{0}-1\right)$ & $0.01 /\left(h_{0}-1\right)$ \\
\hline
\end{tabular}

当 $K, N, L$ 取不同值时, 文献 [10]对MSDD的检测性已 经进行了详细的分析, 因此, 本文主要对不同MDS模型、 散射点个数以及散射点个数估计失配时对MSDD和AMSDD检 测性能的影响, 不失一般性, 假设 $K=15, N=2, L=1, P_{\mathrm{fa}}=10^{-4}$ 。 信杂比定义为 $[10]$ :

$$
\mathrm{SCR}=\left(\mathrm{p}^{\mathrm{H}} \Sigma^{-1} \mathrm{p}\right) \sigma_{s}^{2} / \sigma_{c}^{2}
$$

当 $K=15, P_{\mathrm{fa}}=10^{-4}$ 时, 表 2 给出了 $h_{0}$ 取不同值时的MSDD 检测阈值。

表2 MSDD的检测阈值， $K=15, \quad P_{\mathrm{fa}}=10^{-4}$ 。

\begin{tabular}{llllll}
\hline$h_{0}$ & 1 & 2 & 3 & 4 & 5 \\
$T$ & 23.837 & 32.506 & 39.190 & 44.624 & 49.152 \\
$h_{0}$ & 6 & 7 & 8 & 9 & 10 \\
$T$ & 52.971 & 56.204 & 58.938 & 61.231 & 63.130 \\
$h_{0}$ & 11 & 12 & 13 & 14 & 15 \\
$T$ & 64.668 & 65.871 & 66.758 & 67.343 & 67.633 \\
\hline
\end{tabular}

分别采用表1中的 4 种MDS模型, 图1 图4给出了目标散 射点个数估计值 $h_{\mathrm{e}}$ 对MSDD检测性能的影响并与AMSDD进行 了比较, 其中, $h_{0}=3$ 。结果表明: 目标能量均匀分布 (Mode1. 1) 时, MSDD和AMSDD的检测性能最好, 目标能量越集中, 检测 性能越差。对于不同的目标模型, MSDD当散射点个数估计 匹配时具有最佳的检测性能, 而当散射点个数估计失配时 存在一定的信杂比损失, 特别是当散点个数估计值小于实 际值时信杂比损失比较严重, 然而, 这一现象随着散射点 能量向单个距离单元集中时得到改善。由图4可以看出, 即 使 $99 \%$ 的能量集中于一个单元时, 当散射点个数估计值小于 实际值时, 也会出现较大的信杂比损失。对于表 1 给出的四 种目标模型, 由图1 图4可以看出, AMSDD不需要事先知道 目标散射点个数, 以及散射点的强弱关系, 而其检测性能 要优于MSDD散射点个数估计匹配时的检测性能。

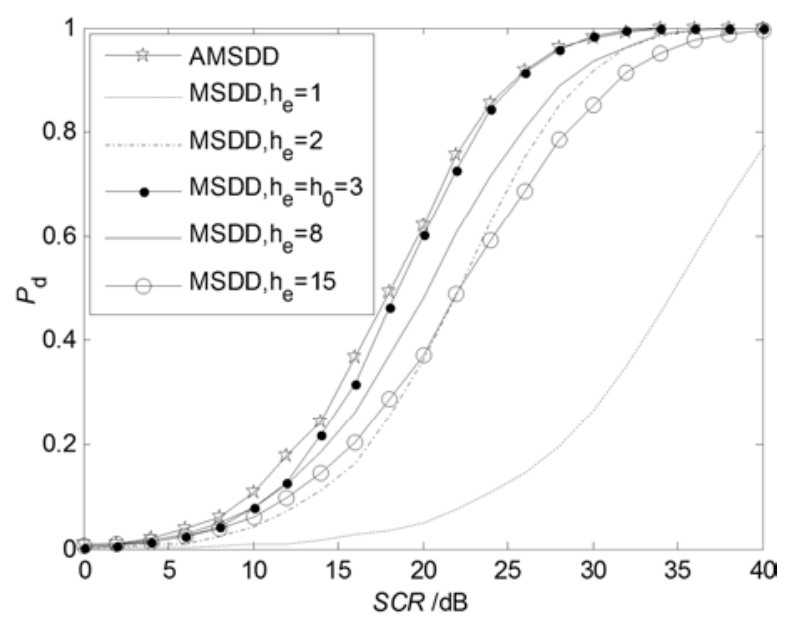

图1 检测器对Mode1. 1的检测性能曲线。 


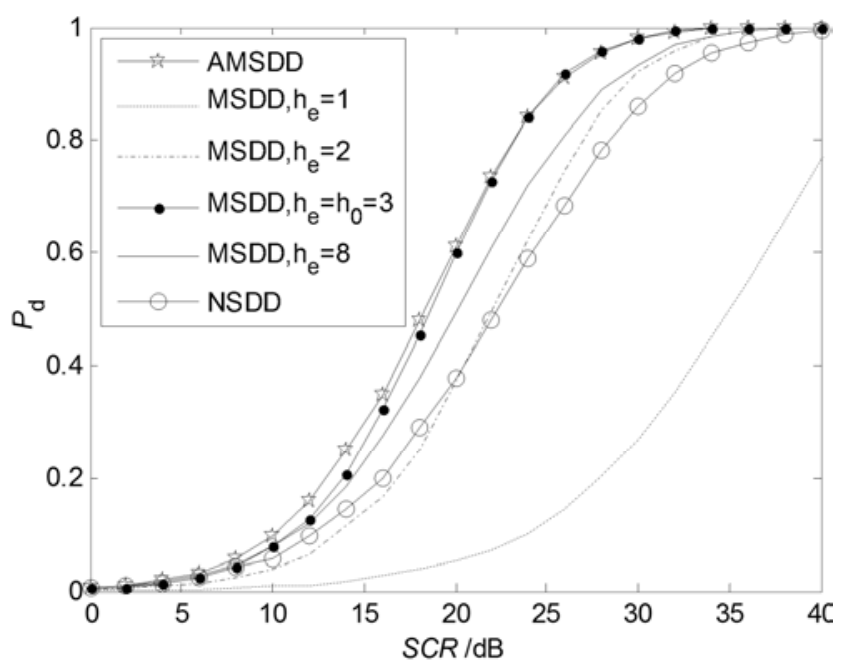

图2 检测器对Mode1.2的检测性能曲线。

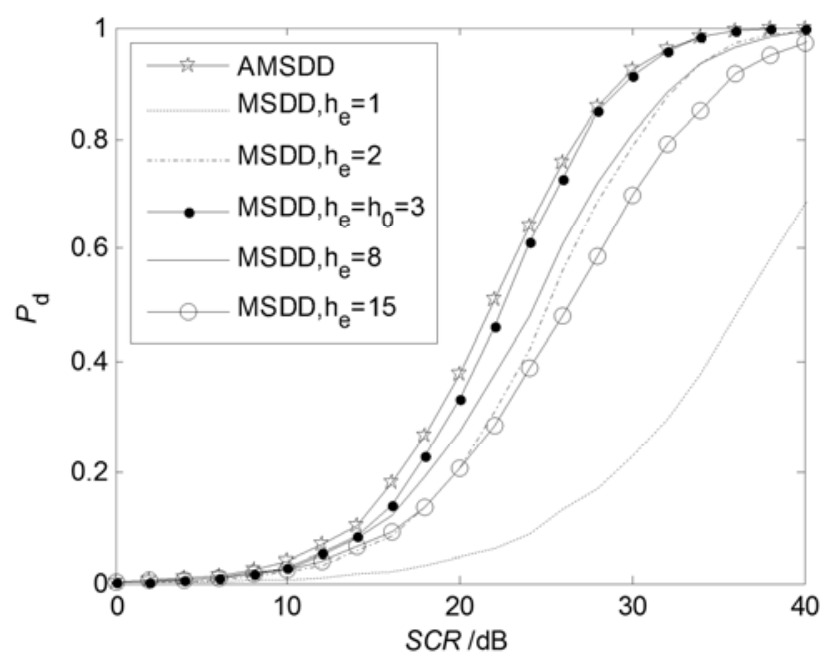

图3 检测器对Mode1.3的检测性能曲线。

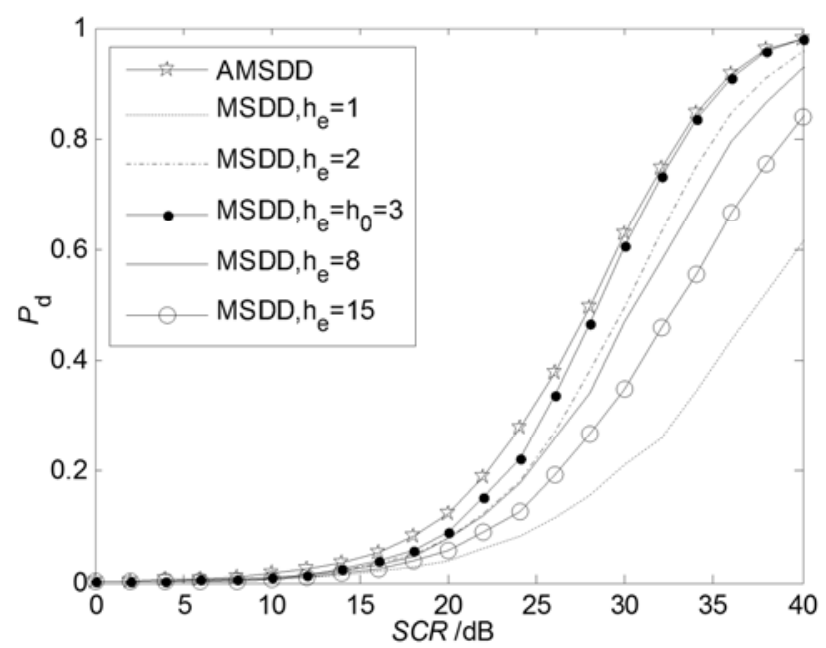

图4 检测器对Mode1.4的检测性能曲线。

图5和图6针对Mode1.1分析了MSDD在散射点个数估计 失配时的检测性能, 并与AMSDD进行比较。图5给出了 $h_{0}=3$, $S C R=20$ 时, $P_{\mathrm{d}}$ 与 $h_{e}$ 的关系曲线。从图 5 可以看出, 当散射点
个数估计值小于目标实际散射点个数时, MSDD存在严重的 检测损失, 这与图1 图4的结果是一致的; 当散射点个数 估计值略大于实际值时, MSDD的检测性能略优于散射点个 数估计匹配时的检测性能, 目标散射个数为其它值时也有 类似的结果 (限于篇幅, 这里没有给出仿真结果); 散射点 个数估计值继续增大, MSDD的检测性能下降, 当 $h_{\mathrm{e}}=K$ 时, $\mathrm{MSDD}$ 就等价为NSDD。图6给出了 $h_{\mathrm{e}}=3, \mathrm{SCR}=10$ 时, $P_{\mathrm{d}}$ 与 $h_{0}$ 的 关系曲线。从图6可以看出, 当 $h_{0}>h_{\mathrm{e}}$ 时, MSDD的检测性能 明显不如AMSDD和SDD。从图5和图6可以看出, AMSDD在目 标散射点稀疏时具有最佳的检测性能, 目标散射点密集时 具有较好的鲁棒性。

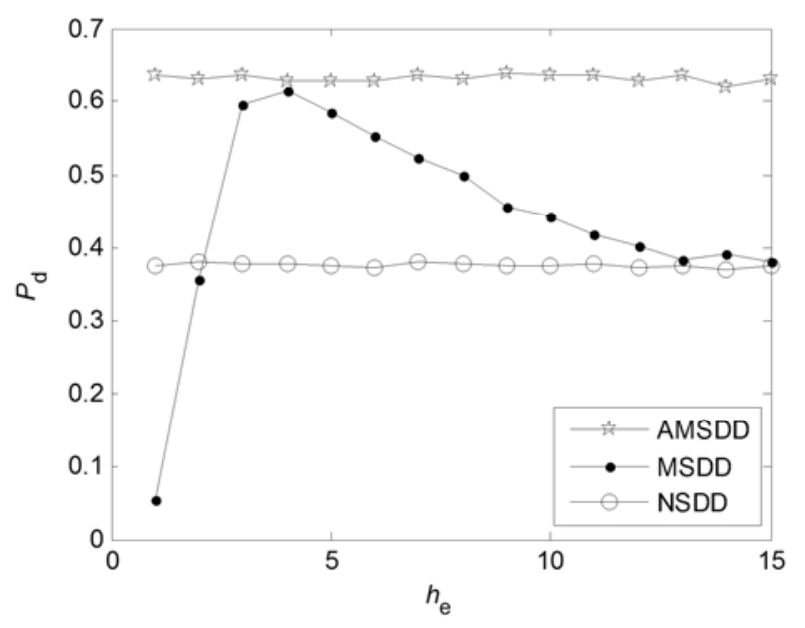

图5 $P_{\mathrm{d}}$ 与 $h_{\mathrm{e}}$ 的关系曲线, $h_{0}=3, \mathrm{SCR}=20$ 。

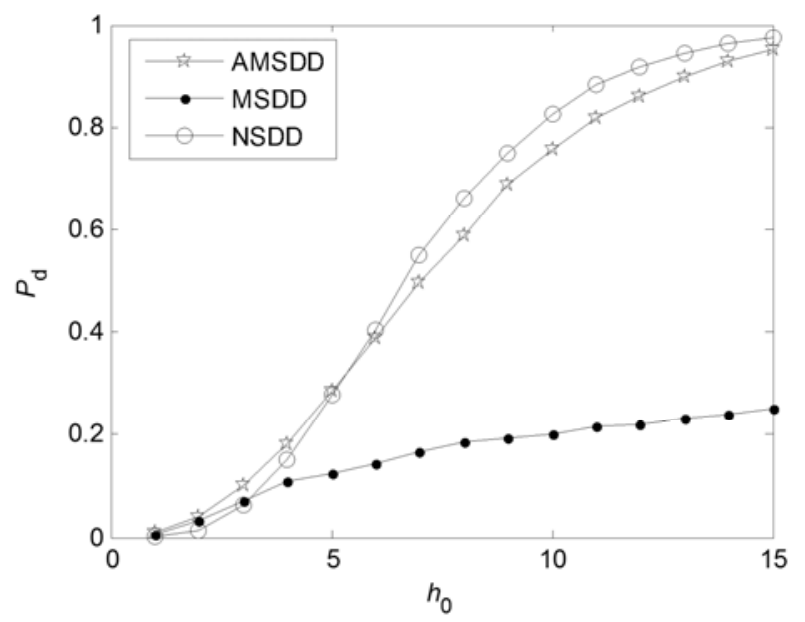

图6 $P_{\mathrm{d}}$ 与 $h_{0}$ 的关系曲线, $h_{\mathrm{e}}=3, \mathrm{SCR}=10$ 。

\section{5. 结论}

本文研究了SIRV非高斯杂波背景下的距离扩展目标 检测问题。针对MSDD检测器在估计目标散射点个数估计失 配时存在一定检测损失的问题，提出了一种能自适应估计 目标散射点个数的MSDD检测器 (AMSDD)。针对不同的MDS 目标模型分析了MSDD在散射点估计匹配和失配时的性能, 并和AMSDD比较, 结果表明MSDD得检测性能随着目标能量 向单个距离单元的集中而下降, 并得出, 即使 $99 \%$ 的能量 
集中于一个单元时, 当散射点个数估计值小于实际值时, 也会出现较大的信杂比损失, 而AMSDD对于不同的MDS目标 模型都具有较好的检测性能。最后, 对MSDD的失配性能进 行了分析, 并与AMSDD和NSDD进行比较, 结果表明, MSDD 在散射点个数估计小于实际值时存在严重的检测损失, 散 射点个数估计大于实际值时的检测性能要优于NSDD, 其中, 略大于实际值时能获得比估计匹配时更优的检测性能, 而 AMSDD在目标散射点稀疏时具有最佳的检测性能, 在目标 散射点密集时具有较好的鲁棒性。需要指出的是, 虽然 AMSDD 比MSDD具有更好的检测性能和鲁棒性, 但也增加了 一定的计算复杂度, 如何在检测性能和计算复杂度之间获 得最优平衡是下一步需要研究的问题。

\section{参考文献}

[1] Wehner D R. High-Resolution Radar, 2nded. Boston, MA: Artech House, 1995.

[2] 简涛, 何友, 苏峰, 等. 非高斯杂波下距离扩展目标检测 器的失配性能分析 [J]. 电子学报, 2010, 38(7): 1478-1482。

[3] Hughes P K. A high-resolution radar detection strategy $[\mathrm{J}]$. IEEE Trans Aerospace Electron System, 1983, (19): 663-667.
[4] Gerlach K, Steiner M J. Adaptive detection of range distributed targets[J]. IEEE Transactions on Signal Processing, 1999, 47 (7): 1844-1851.

[5] Gerlach K, Steiner M, Lin F C. Detection of a spatially distributed target in white noise [J]. IEEE Signal Processing Letters, 1997, 4(7): 198-200.

[6] Conte E, De Maio A, Ricci G. GLRT-based adaptive detection algorithms for range-spread targets [J]. IEEE Transactions on Signal Processing, 2001, 49(7): 1336-1348.

[7] 戴奉周, 刘宏伟, 吴顺君. 一种基于顺序统计量的距离扩 展目标检测器 $[\mathrm{J}]$. 电子与信息学报, 2009, 31(10): 2488-2492。

[8] Rangaswamy. M, Weiner. D. D and Ozturk. A. Computer generation of correlated Non-Gaussian radar clutter [J]. IEEE Transactions on Aerospace and Electronic Systems, 1995, 31(1): 106-116.

[9] K. Gerlach. Spatially distributed target detection in non-Gaussian clutter [J]. IEEE Transactions on Aerospace and Electronic Systems, 1999, 35(3): 926-934.

[10] 简涛, 何友, 苏峰, 等. 非高斯杂波下修正的SDD距离扩 展目标检测器 $[J]$. 电子学报, 2009, 37 (12)：2662-2667。

[11] Strong Scatterers Integrator Based on ADT in Non-Gaussian Cluter. Gu X. F., Hao X. L., Yang G. L. et.al. Science Discover, 2016, vol 4(1):26-30. 